\title{
Laser Irradiated Foam Targets: Absorption and Radiative Properties
}

\author{
Martina Salvadori ${ }^{1,2, \star}$, Pier Luigi Andreoli ${ }^{2}$, Mattia Cipriani ${ }^{2}$, Fabrizio Consoli ${ }^{2}$, Giuseppe Cristofari $^{2}$, Riccardo \\ De Angelis ${ }^{2}$, Giorgio di Giorgio ${ }^{2}$, Danilo Giulietti ${ }^{1}$, Francesco Ingenito ${ }^{2}$, Sergey Yu. Gus'kov ${ }^{4}$, and Alexander A. \\ Rupasov $^{3}$ \\ ${ }^{1}$ Pisa University, Physics Department "E.Fermi" and INFN \\ ${ }^{2}$ ENEA Fusion and Nuclear Safety Department, C.R. Frascati, Via E. Fermi 45, 00044 Frascati, Italy \\ ${ }^{3}$ Lebedev Physical Institute, Russian Accademy of Sciences, Moscow \\ ${ }^{4}$ National Research Nuclear University, Moscow Engineering Physics Institute MEPhl
}

\begin{abstract}
An experimental campaign to characterize the laser radiation absorption of foam targets and the subsequent emission of radiation from the produced plasma was carried out in the ABC facility of the ENEA Research Center in Frascati (Rome). Different targets have been used: plastic in solid or foam state and aluminum targets. The activated different diagnostics allowed to evaluate the plasma temperature, the density distribution, the fast particle spectrum and the yield of the X-Ray radiation emitted by the plasma for the different targets. These results confirm the foam homogenization action on laser-plasma interaction, mainly attributable to the volume absorption of the laser radiation propagating in such structured materials. These results were compared with simulation absorption models of the laser propagating into a foam target.
\end{abstract}

\section{Introduction}

Porous materials of light elements consist of randomly distributed solid fibers or membranes, with transversal size $b_{0}$ in the range of few microns up to hundreds of microns, separated by voids of similar dimensions. According to their pore size $\delta_{0}$, these materials can be divided in two groups, large pore foams and small pore foams. The first one comprehends porous plastics $\left([\mathrm{CH}]_{n},\left[\mathrm{CH}_{2}\right]_{n}\right)$, porous Beryllium and agar-agar $\left(\mathrm{C}_{12} \mathrm{H}_{18} \mathrm{O}_{9}\right)$, with pore size and thickness of solid elements ranging from $30 \mu \mathrm{m}$ to $100 \mu \mathrm{m}$ and from 0.1 to $1 \mu \mathrm{m}$ respectively; cellulose triacetate $\left(\mathrm{C}_{12} \mathrm{H}_{16} \mathrm{O}_{8}\right)$ and trimethylolpropane triacrylate $\left(\mathrm{C}_{15} \mathrm{H}_{20} \mathrm{O}_{6}\right)$ are in the small pore group, with pore size ranging from 1 to $10 \mu \mathrm{m}$ and the thickness of solid elements from 0.01 to $0.1 \mu \mathrm{m}$.

The average density of foams can vary from few $\mathrm{mg} / \mathrm{cm}^{3}$ to several hundreds. This wide variety in characteristic dimensions leads to different behavior under laser irradiation. In particular, according to their initial average density $\rho_{a}$, corresponding to an electron density $n_{e}=\frac{\rho_{a} Z_{e f f}}{M}$ where $Z_{\text {eff }}$ and $M$ are respectively the effective state of charge and the mass of the atoms or molecules, and to the impinging laser wavelength $\lambda$, we can distinguish overcritical foams, characterized by $n_{e}$ greater than the critical density $n_{c r}$, from subcritical foams, with $n_{e}<n_{c r}$, where

$$
n_{c r}=\frac{1.1 \times 10^{21}}{\lambda_{\mu \mathrm{m}}^{2}} \mathrm{~cm}^{-3}
$$

\footnotetext{
^e-mail: martina.salvadori90@gmail.com
}

Regardless of this distinction, due to the internal structure of the material, at the initial stage of interaction, the laser radiation has the chance to penetrate up to a distance in both cases defined by the initial transparency length:

$$
L_{0} \simeq C\left(\frac{\rho_{s}}{\rho_{a}}\right)^{\frac{1}{5}} \delta_{0}
$$

where $\rho_{s}$ is the density of the solid elements and $\mathrm{C}$ is a constant which depends on their shape [1]. For our foam parameters, $\delta=40 \mu \mathrm{m} \rho_{a}=10 \mathrm{mg} / \mathrm{cm}^{3}, \rho_{s}=1050 \mathrm{mg} / \mathrm{cm}^{3}$, the estimated initial transparency length is equal to 500 $\mu \mathrm{m}$.

Laser irradiation gives rise to an homogenization process leading to a fully homogeneous plasma. As the process goes on, the transparency length changes accordingly to density variations. In this phase laser radiation is absorbed, via inverse Bremsstrahlung mechanism, in a plasma of stochastically varying density created by the expansion of the solid parts under laser irradiation. The volume of overcritical regions increases, producing a progressive decrement in the depth of the absorption region. In both cases laser light absorption continues according to usual mechanisms for a homogeneous material. In overcritical foams the radiation is absorbed in the subcritical plasma developed in front of the target. In subcritical foams laser radiation is absorbed behind the ionization front propagating inside the target.[2].

These considerations show that the initial homogenization stage has a peculiar role in laser light absorption, allowing a deeper penetration in the target even with an overcritical average density. This explains the experimental observation of a deep absorbing region and an anoma- 
lously high absorption efficiency for overcritical foams up to $80 \%-90 \%$ of incoming radiation [3]. Moreover, these peculiarities lead to some interesting properties such as the reduction of the irradiation inhomogeneities [4] and the amplification of ablation pressure [5].

For all these reasons, the foams are of great interest for their applications in Inertial Confinement Fusion experiment, as they are excellent absorbers of laser radiation and inhibitors of detrimental hydrodynamic instabilities. The aim of this work is to collect further information about three aspects of the laser-foam interaction: The plasma density profile, the absorption mechanism and the emitted radiation. In this paper we present the main results obtained irradiating overcritical porous Polystyrene comparing them with the one obtained by the irradiation of solid Polystyrene and Aluminum.

\section{Experimental setup}

The experiments have been performed at ABC Laser, a $\mathrm{Nd}$ laser with a wavelength $\lambda=1054 \mathrm{~nm}$, a pulse energy $\simeq 40 \mathrm{~J}$, a time duration $\tau_{L} \simeq 3 \mathrm{~ns}$ with a rising time of about 1.5ns. An $F=1$ optics allows to focus the laser beam down to $\sim 100 \mu \mathrm{m}$ spot diameter, corresponding to an intensity on target of $10^{13} \mathrm{~W} / \mathrm{cm}^{2}$. The targets were made of solid Polystyrene with density $\rho=1050 \mathrm{mg} / \mathrm{cm}^{3}$ and thickness $\delta=1100 \mu \mathrm{m}$; solid Aluminum with $\rho=2700$ $\mathrm{mg} / \mathrm{cm}^{3}$ and $\delta=510 \mu \mathrm{m}$; different porous polystyrene targets with thickness ranging from 290 to $915 \mu \mathrm{m}$ and $\rho_{a}=10 \mathrm{mg} / \mathrm{cm}^{3}$. Because of their density the foam samples are overcritical for ABC laser wavelength.

The main diagnostics in use were devoted to the investigation of density profile and radiation emission. For this purpose we use a Nomarski interferometer, an optical streak camera, silicon surface barrier detectors and diamonds detectors. The diagnostic layout is shown in figure 1. A frequency doubled diagnostic beam is employed in

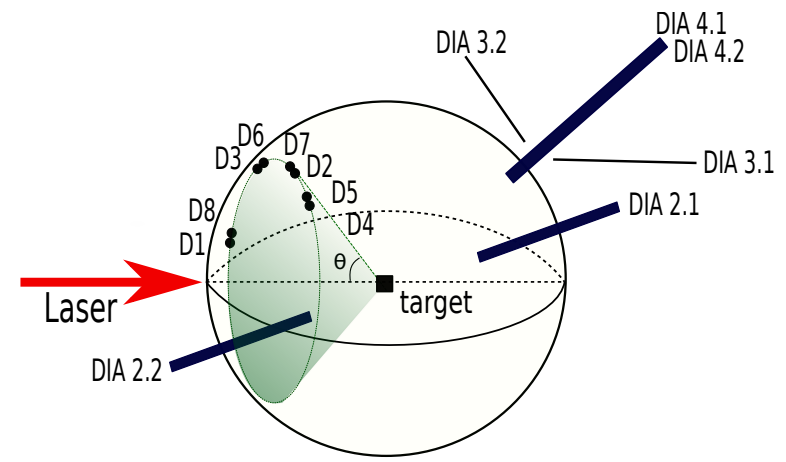

Figure 1: Experimental chamber layout, Vacuum diodes are placed on the base circumference of a cone whose vertex is on target. Thanks to the symmetry in the plasma radiation emission, these positions ensure to have comparable signals on each diode. Diamonds are placed at different distances and angles with respect to the target.

the Nomarski Interferometer which provides information about the electron density profile of the generated plasma.
The optical streak camera allows to detect the plasma expansion normal to the target surface, with a temporal and spatial resolution of $20 \mathrm{ps}$ and $20 \mu \mathrm{m}$ respectively.

The emitted X-Ray radiation is observed through Diamond detectors, which are sensible to wavelength shorter than 250nm, and through silicon surface barrier detectors sensible also to visible light. Since we are interested in higher energy emission, we used Beryllium and Nickel filters of various thickness to select the proper spectral region. Diamond detectors were also employed for Time Of Flight (TOF) measurements. In table 1 the distances of the diamonds from the target and the diode's filters layout are reported.

\begin{tabular}{|c|c|}
\hline Diode & Filter $(\mu \mathrm{m})$ \\
\hline D1 & Be 25.4 \\
\hline D2 & Ni 10.91 \\
\hline D3 & $\mathrm{Ni} 4.59$ \\
\hline D4 & Ni 7.61 \\
\hline D5 & Ni 9 \\
\hline D6 & Be 50.8 \\
\hline D7 & Ni 5.46 \\
\hline D8 & Be 12.7 \\
\hline \multicolumn{2}{|r|}{ (a) } \\
\hline Diamond & Distance $(\mathrm{mm})$ \\
\hline DIA 2.1 & 1130 \\
\hline DIA 2.2 & 1120 \\
\hline DIA 3.1 & 899 \\
\hline DIA 3.2 & 899 \\
\hline DIA 4.1 & 1739 \\
\hline DIA 4.2 & 1739 \\
\hline
\end{tabular}

Table 1: (a)Filtering layout of vacuum diodes. (b) Diamond detectors distances from target

\section{Experimental Results and Discussion}

\section{Density Profile}

Images obtained with Nomarski interferometer are shown in figure 2. The interference pattern gives no evidence of a sizable difference in the electron density distribution between the solid and the foam targets. Most probably this is due to the fringe blurring produced in the region of interest by the relative long pulse duration of the probe. Nevertheless this diagnostic channel provide an indirect information about the density gradients. In fact comparing the three images it is possible to see that in the case of irradiation of solid polystyrene and solid Aluminum the presence of second harmonic emission is apparent, meanwhile it is completely absent in the case of irradiation of porous 


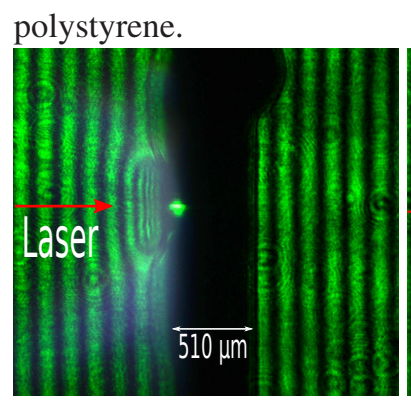

(a)

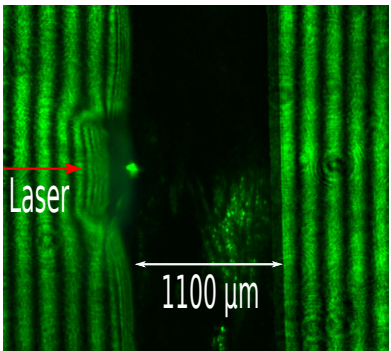

(b)

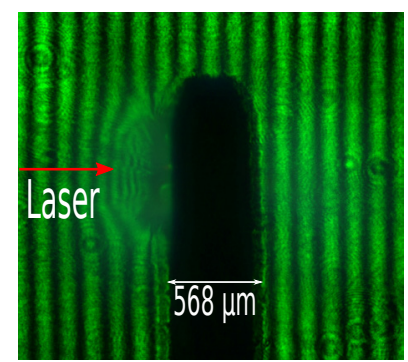

(c)

Figure 2: Images obtained by the Nomarski interferometer when irradiating target of different materials: (a) Aluminum $510 \mu \mathrm{m}$ thick (b)Solid Polystyrene $1100 \mu \mathrm{m}$ thick and (c)Porous Polystyrene $568 \mu \mathrm{m}$ thick

Since the second harmonic emission is related to the presence of plasma density gradients [6],

$$
\mathbf{J}(2 \omega)=\frac{i n_{e} e^{2}}{4 m^{2} \omega^{3}} \nabla(\mathbf{E} \cdot \mathbf{E})+\frac{i e^{2}}{m^{2} \omega^{3}}\left[\frac{\nabla n_{e} \cdot \mathbf{E}}{1-\omega_{p}^{2} / \omega^{2}}\right] \mathbf{E}
$$

where $m$ is the electron mass, $n_{e}$ is the plasma electron density, $e$ is the electron charge, $\omega$ is the frequency of the laser, $\omega_{p}$ is the plasma frequency and $\mathbf{E}$ is the electric field, its absence can be related to small value of $\nabla n_{e}$, hence to the production of a plasma characterized by a smoother density profile. For this reason we can assume that the irradiation of porous polystyrene produces a plasma whose density variations are smoother than in the case of irradiation of solid polystyrene and aluminum.

Another indirect evidence of plasma homogeneity comes from time of flight measurement. As we can see in figure 3 the diamond signals are characterized by a first higher peak, which is generated by radiation, and, at later times, by a series of subsequent lower peaks. These are ascribable to the incoming accelerated particles, in particular to protons and ions. The force providing ions acceleration arises from the electric field generated by the displacement of electrons which are accelerated trough the electron plasma waves. The subsequent velocity acquired by a charged particle is given by the equation 4 .

$$
\mathbf{v}(t)=\left(\frac{2 \mathbf{E} e L_{a c c}}{m_{p}} \frac{Z}{A}\right)^{1 / 2}=\mathbf{v}_{p}\left(\frac{Z}{A}\right)^{1 / 2}
$$

where $L_{a c c}$ is the length in which the accelerating force acts, $m_{p}$ the proton mass, $Z$ and $A$ are the state of charge and the number of mass of the ions respectively, $v_{p}$ is the velocity acquired by a proton.

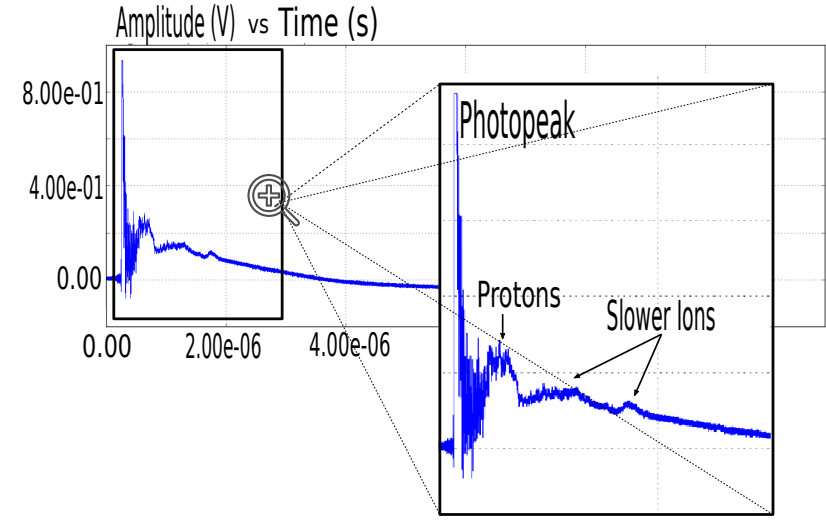

Figure 3: Typical signal obtained from diamond detectors and a magnification of its most interesting part. In order to avoid the dependence of time measurement from the delay of the oscilloscope, we took as reference the photopeak time and we have measured the time interval $t_{i}-t_{\gamma}$ where $t_{i}$ and $t_{\gamma}$ are the time at which ions and photons arrive on the diamond detectors.

From the equation 4 it is possible to see that the fastest particles will be those with a larger $\frac{Z}{A}$ ratio, hence we will detect first protons and than ions. In our experimental set up fast electrons signal is superposed with the one given by the photopeak. From these considerations we are able to identify in figure 3: the photopeak, the protons and the different ions of the target specimen. Comparing the results obtained for the velocity of protons generated by different materials, it turns out that irradiation of porous polystyrene produces slower ions than the irradiation of solid aluminum or polystyrene, as it is possible to see in figure 4 . Since in our simple scheme, ac-

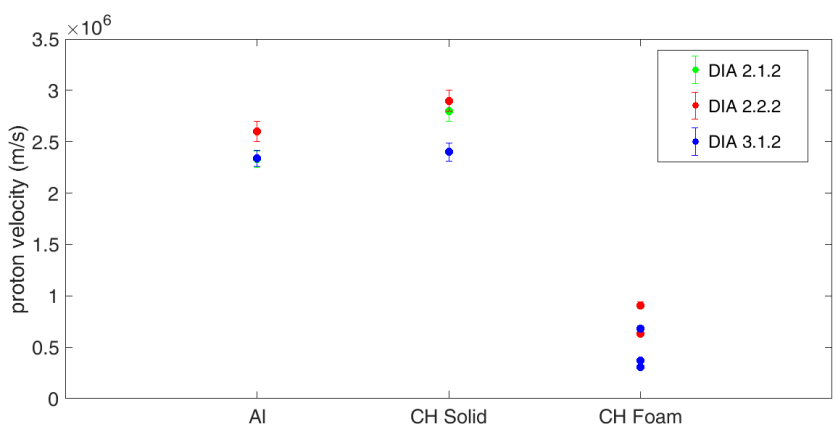

Figure 4: Velocities of protons generated by irradiation of different types of material. The velocities are obtained trough Time of Flight measurement performed with Diamond detectors.

celeration of ions is related to the amount of accelerated electrons, less energetic ions means a lower yield of accelerated electrons. In our experimental condition the main mechanism of electron acceleration is related to the presence of electron plasma waves produced by parametric instabilities which, to be generated, require a threshold laser 
intensity that could be reached by the activation of the filamentation instability. Since our irradiation parameters are under this threshold value, we think that in the case of solid material the phenomenon of filamentation is activated by the presence of inhomogeneities in laser irradiation (i.e. due to the presence of the hot spots) and contributes to reach the intensity threshold, while in the case of porous materials filamentation doesn't occur because of the homogenizing action of foams on laser irradiation inhomogeneities. In addition to intensity gradient, filamentation is facilitated also by the presence of high density gradients hence its absence can be related to the production of a plasma characterized by smoother variation in density [7].

\section{Absorption and Emission Processes}

Through the images obtained by the streak camera it has been possible to investigate on plasma emission. In figure 5 shots on polystyrene foams of different thickness are reported. The luminescence at the front side of the target arises from the plasma self emission and allow to follow its expansion. The luminescence at the rear side is due to the plasma generated by the hydrothermal wave propagating inside the target.

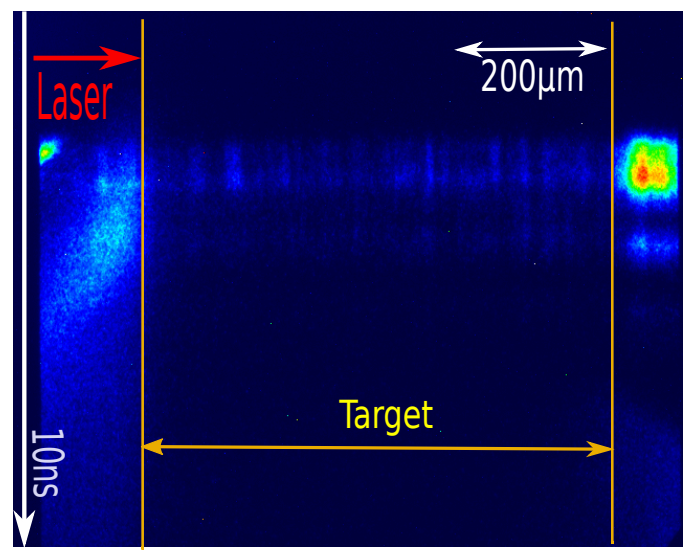

(a)

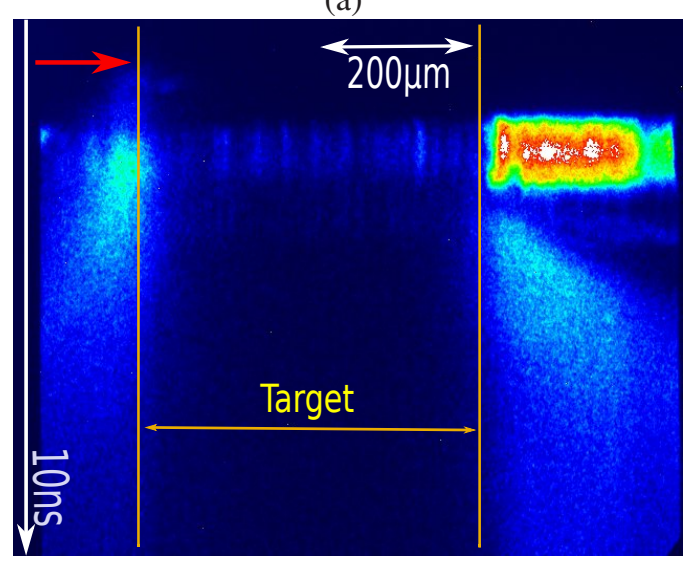

(b)

Figure 5: Images obtained by the streak camera in the case of irradiation of Porous polystyrene with thickness (a) about the initial transparency length $(430 \mu \mathrm{m})$ and (b)lower than the initial transparency length $(330 \mu \mathrm{m})$
Streak camera images provides another useful information, in fact focusing the laser near to the external surface toward the streak camera direction, as shown in figure 6(a), it is possible to observe the emission coming from the internal region of the porous target as the laser propagates inside it, figure 6(b). This allows us to perform measurement of its propagation velocity. We obtain values consistent

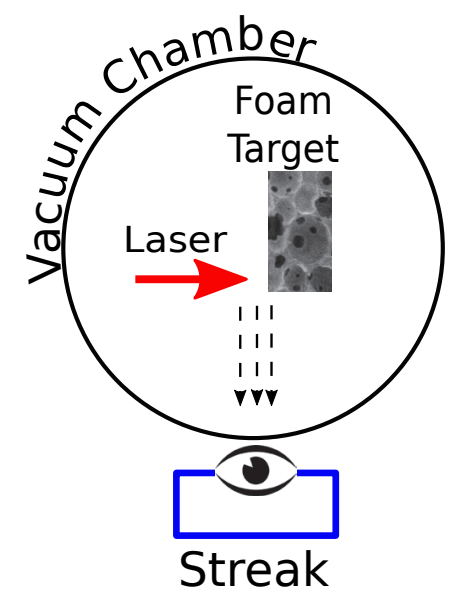

(a)

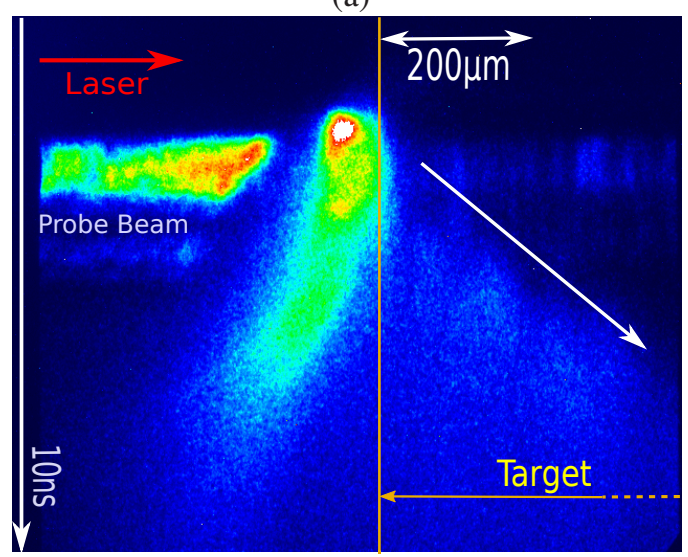

(b)

Figure 6: (a) Top view of the focusing scheme during the shot. (b) Image obtained from the optical streak camera, The white arrow highlights the propagation of ionization wave inside porous polystyrene. From its slope it is possible to evaluate the propagation velocity.

with theoretical simulation, $v=(1.2 \pm 0.1) \times 10^{7} \mathrm{~cm} / \mathrm{s}$. A more accurate survey of the images in figures 5 and 6 provides information about the expansion velocity of plasma, that is of the order of the sound velocity in the medium given by the relation:

$$
c_{s}=3 \times 10^{7} \sqrt{\frac{Z T_{e}(\mathrm{KeV})}{A}} \frac{\mathrm{cm}}{\mathrm{s}} .
$$

From this value it is possible to estimate the electron plasma temperature. As shown in figure 7, comparing the results for different materials, Porous Polystyrene shows higher temperature than solid one. This result suggest a greater emission of radiation at higher energy, to investigate this aspect we examine the data obtained from diamonds and silicon surface barrier detectors. The first, sig- 


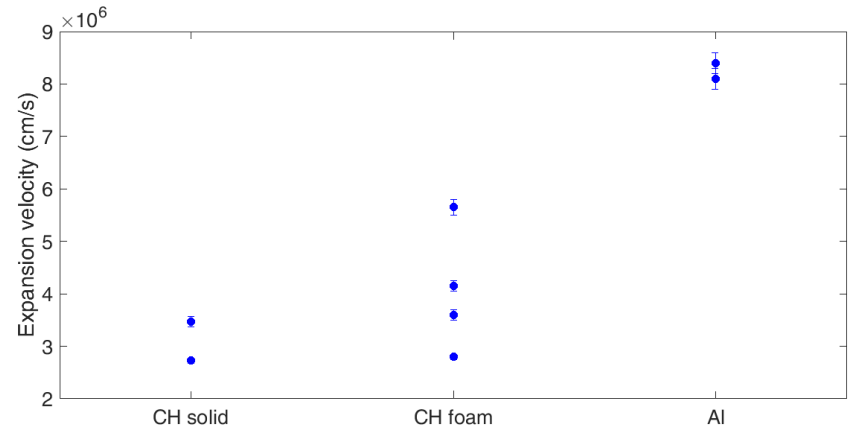

Figure 7: Plasma expansion velocity measurement obtained by streak camera images

nal is reported in figure 8, show an higher photopeak amplitude in the case of porous polystyrene than in the one of solid plastic, and similar photopeak amplitude for Aluminum and porous Polystyrene targets. Solid and Porous

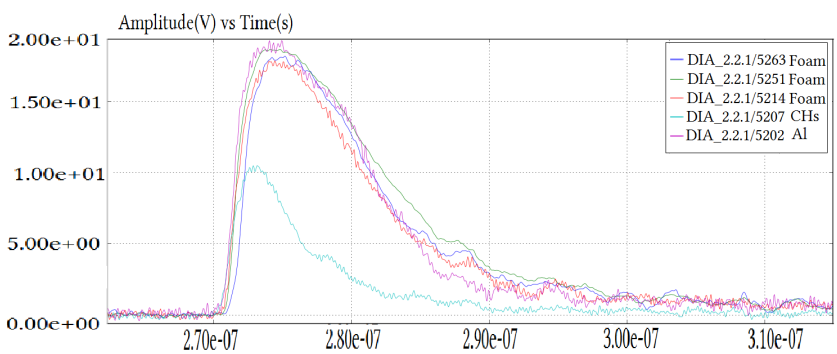

Figure 8: Photopeak amplitude obtained irradiating different types of material

polystyrene have the same chemical composition, hence this high difference in the emission behavior has to be attribute to their different internal structure. Since an higher emission is related to an higher absorption this result is consistent with the described peculiar absorption process. Due to the composition of plastic material we expect that the main contribution to the radiation emission comes from Bremsstrahlung process, while in the case of Aluminum both contribution of continuum and lines emission play a central role. Moreover we know that the contribution of Bremsstrahlung is proportional to the atomic number of the emitting element, from these consideration, in the case of solid material, it is evident the result shown in figure 8 . On the other hand we suggest that the similar photopeak amplitude for Aluminum and porous polystyrene can be another evidence of an higher absorption in the case of porous materials.

Vacuum diodes confirm that irradiation of porous plastics provides an higher radiation emission than in the case of interaction with solid one. As it is possible to see in figure 9, this result is apparent when we consider the signals obtained from the diodes filtered with the thickener Nickel foil, whilst if we look at data obtained from diodes with a lower filtering, the differences in the amount of emitted radiation gradually disappears. Since an higher value of filtering allows only to higher energy radiation to be detected, this result suggest that the differences in emission of radiation arises from the high frequency region, which means that in the case of porous polystyrene we are dealing with an hotter plasma than in the case of solid polystyrene, result which is consistent with streak camera measurement.

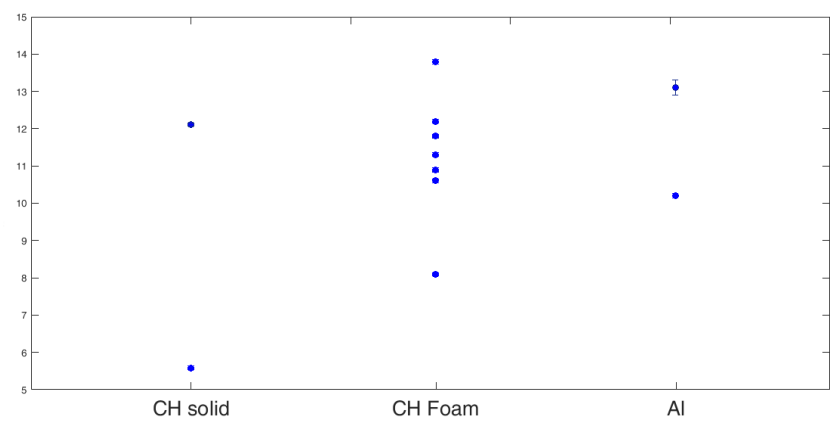

(a)

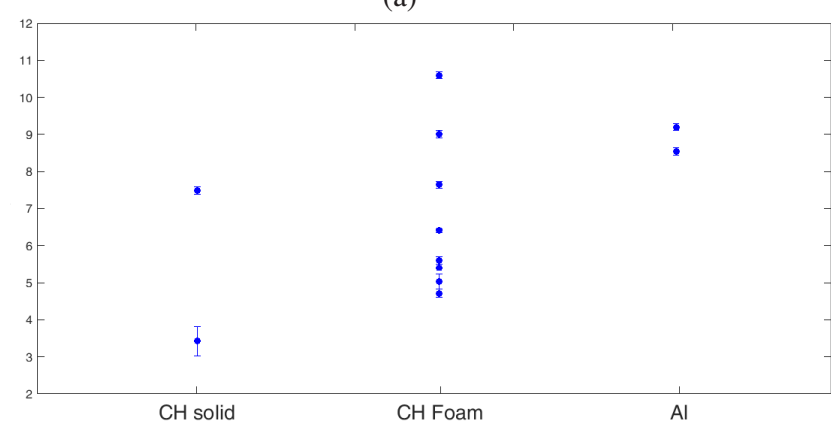

(b)

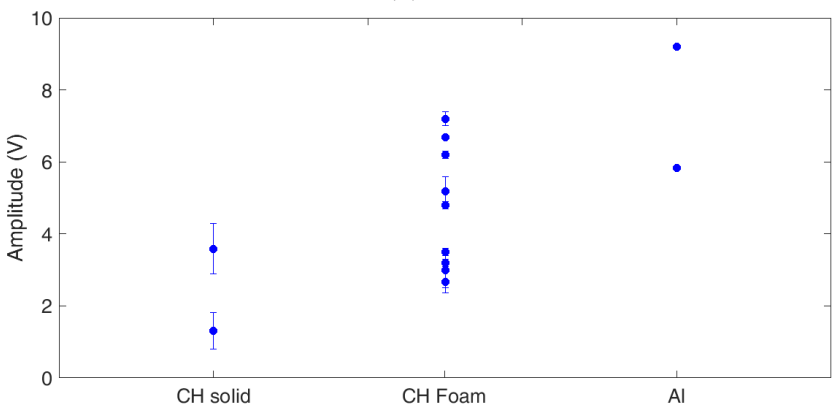

(c)

Figure 9: Comparison of the amplitudes of vacuum diodes signal when irradiating different types of material. Each graph is obtained with a different thickness of Nickel filter: (a) $4.59 \mu \mathrm{m}$, (b) $9 \mu \mathrm{m}$ and (c) $10.91 \mu \mathrm{m}$.

\section{Conclusion}

During our experiments we investigated the absorption and emission properties of targets of different chemical composition and internal structure. In particular, in foam targets, we highlighted an improvement of the laser radiation absorption and the subsequent larger X-Ray emission. Furthermore indirect measurements suggest that the irradiation of porous materials produces a more homogeneous plasma than the irradiation of solids.

This work was supported by the Program of Increase in the Competitiveness of the National Research of Nuclear 
University MEPhI. The work of S. Gus'kov and A. Rupasov was also supported by Russian Foundation for Basic Research project No. $17-02-00059-$ a. The experimental campaign was carried out at ENEA research centre in Frascati, in The $\mathrm{ABC}$ laboratory.

\section{References}

[1] S. Gus'kov, Journal of Russian Laser Research 31, 574 (2010)

[2] S. Gus'kov, M. Cipriani et al., Plasma Physics and Controlled Fusion 57, 125004 (2015)
[3] A Bugrov, S. Gus'kov and V Rozanov, Journal of experimental and theoretical physics 88, 441 (1999)

[4] S. Gus'kov et al., Laser and Particle Beams 18, 1 (2000)

[5] R. De Angelis et al., Physics of Plasma 22, 072701 (2015)

[6] Y. R. Shen, The Principles of Nonlinear Optics (John Wiley and Sons, New York, 1984) 10

[7] D. Giulietti and L. Gizzi, Nuovo Cimento, 21, 1-93, (1998) 